exclusion chromatography of biopolymers in chapter 7, the treatment is in some respects misleading. For example, in the case of the gel media used with biopolymers, there is an increased zone dispersion, which has a good theoretical foundation in the polydispersity of retention sites (that is pores) in the stationary phase. This problem has been extensively discussed by Ackers and others. The consequent poor resolution has lead to the virtual abandonment of exclusion chromatography for the determination of the molecular weights of biopolymers by biochemists, who now favour electrophoresis in a continuous molecular sieving medium for this purpose. Also contrary to the impression given on $\mathrm{p} 76$, adsorption problems are still severe with many proteins on inorganic exclusion media. Polymer chemists seem to be indeed fortunate in the exclusion media available to them.

Chapters $8-10$ give a very good account of the hardware of HPLC, columns, injection systems, pumps and detectors. Perhaps uniquely, this section also includes a discussion on the use of postcolumn reactor systems, which may be increasingly used in the future to increase both sensitivity and selectivity. Although the authors seem to be optimistic about

\section{Molecular damage after radiation}

Radiation Effects: ESR and ENDOR Analysis. By H. C. Box. Pp. 280. (Academic: New York and London, 1978.) $\$ 24.50$.

THis book describes the molecular damage which occurs in carboxylic acids, amides, amino acids, peptides, proteins, pyrimidines, purines and nucleic acids, when these substances are bombarded with high energy radiation. In addition to the immediate effect of the radiation, the secondary processes are described and some overall mechanisms are followed through. Most of this information is derived from a knowledge of what radicals are formed, in what yield, how quickly they react, and with what. It is the unique contribution of electron spin resonance (ESR) supplemented by ENDOR (a special form of nuclear magnetic resonance which uses the ESR signal as a means of detection), which makes this study possible.

The first five chapters describe the characteristics of the ESR and ENDOR methods. In this part of the book, hyperfine structure and $g$ values are treated with fair rigour and the Hückel and INDO methods of computing spin the future development of many of the presently less common detectors, I feel that the current escalation in the cost of HPLC equipment may put many of these more sophisticated detectors out of the reach of any but specialised laboratories. From this aspect I consider the comparison of analytical methods in Table 11.1 rather biased in favour of HPLC, especially as regards specificity and cost. For example, a good radioimmunoassay can be at least as specific as an HPLC estimation, whereas instrumental costs have been overtaken by inflation during the time taken for publication of the book.

Applications of HPLC, particularly to pharmaceutical and clinical analyses, and a description of the Wolfson departmental course experiments conclude the book. The all-important topic of column packing is excellently covered, although late in the book.

As a comprehensive, modern and methodical treatment of all aspects of HPLC, this book has few rivals, especially at its very modest price.

C. J. O. R. Morris

C.J. O. R. Morris is Ementus Professor of Experimental Biochemistry in the University of London, UK.

densities are compared with each other and with experiment. The next five chapters are concerned with the radiation effects and mechanisms while the last chapter sets all this into perspective with current and projected work on: radiation modifiers which might be used in cancer treatment; conversion of solar energy into chemical energy; the relationship between radiation-induced carcinogenesis and DNA repair processes; and radiation damage in electron microscopy.

In general the book is well balanced between theory and experiment. It is well written and makes a fascinating account of our knowledge of the molecular events which follow irradiation of biological material. There are lots of well labelled chemical formulae and plenty of tables of data for reference purposes in the text and in appendices; these will make the book valuable for active workers in this field. The reviewer personally favours a slightly more experimental approach and would have preferred to see a few more actual spectra, particularly ENDOR spectra and more details of the assignments on these. Space could have been found at the expense of some of the mathematical detail in the early chapters which many readers more familiar with cell biochemistry might find difficult and unnecessary to follow.

J. F. Gibson

J. F. Gibson is Lecturer in Chemistry at Imperial College, University of London, UK.

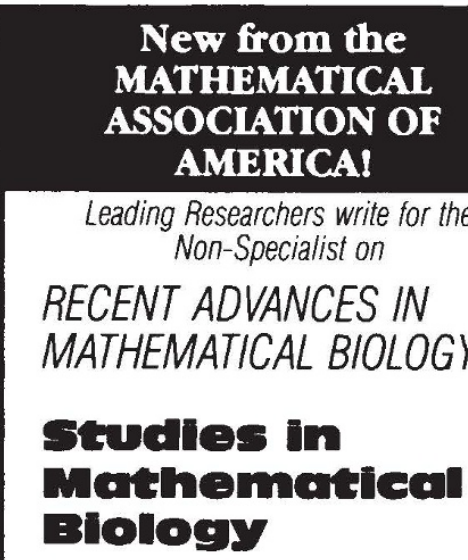

MAA Studies in Mathematics Volumes 15 and 16

Simon Levin, Editor

The widely respected series MAA Studies in Mathematics has long published expository papers on topics at the research frontiers written for a broad mathematical audience. STUDIES IN MATHEMATICAL BIOLOGY expands that tradition; this two-volume set is written also for the mathematically prepared life scientist. Eighteen leading researchers write of recent work in the active field of mathematical applications, providing an authoritative yet refreshingly accessible sampler of advances and current research directions. These new books will be useful for seminars, independent study projects, and supplementary reading for all who are interested in the contributions of mathematics and biology.

Part I, Cellular Behavior and Development of Pattern. Papers by John Rinzel, Jack Cowan and G. B. Ermentrout, Michael Arbib, Lee A. Segel, Nancy Kopell, E. C. Zeeman, Stuart Kauffman, Arthur T. Winfree, J. M. Guckenheimer.

Volume 15 ; xiv +315 pages + index. Hardbound. List $\$ 16.00$.

\section{Part II, Populations and} Communities. Papers by Robert M. May and Robert H. MacArthur, Donald Ludwig, S. I. Rubinow, George F. Oster, Simon A. Levin, W. J. Ewens, Samuel Karlin, Thomas Nagylaki.

Volume $16, x x+308$ pages + index Hardbound. List $\$ 16.00$.

Special price for the two-volume set: $\$ 27.00$

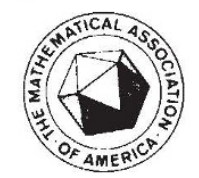

Order your copies now from: MAA, 1529 Eighteenth St., N.w., Washington, D.C. 20036. (Orders from Europe, Africa, Middle East should be sent to: John Wiley and Sons, Ltd., Baffins Lane, Chichester, West Sussex, P019 1UD. England. Prices slightly higher.) 